Supporting Information for:

\title{
Phenylalanine 445 within Oxidosqualene-Lanosterol Cyclase from Saccharomyces cerevisiae Influences C-Ring Cyclization and Deprotonation Reactions
}

Tung-Kung Wu*, Yuan-Ting Liu, Feng-Hsuan Chiu, and Cheng-Hsiang Chang Department of Biological Science and Technology National Chiao Tung University 300, Hsin-Chu, Taiwan (Republic of China)

\section{Materials and methods}

Generation of ERG $7^{F 445 X}$ Site-Saturated Mutants. The plasmid pTKERG7RS314WT was used as a template to construct the $E R G 7^{\mathrm{F} 445 \mathrm{X}}$ site-saturated mutagenesis plasmids. The oligonucleotide primers used were 5'-AGAAAGGGTGCCTGGGGCNNNTCAACA-3' 5'-TGTTGANNNGCCCCAGGCACCCTTTCT-3', which cause the TTC-to-NNN codon change and the GGGGCT-to-GGTGCㅡㅡ changes to generate nearby BanI restriction sites. Site-saturated mutagenic DNAs were constructed by use of PCR amplification with the QuickChange Site-Directed Mutagenesis kit (Stratagene Inc., La Jolla, CA), as described previously. ${ }^{1,2}$ The exact amino acid substitution at Phe445 position was determined by sequence of the DNA by use of an ABI PRISM 3100 auto-sequencer, according to the manufacturer's protocol (Applied Biosystems, Foster City, CA, USA). The recombinant $\mathrm{pERG} 7^{\mathrm{F} 445 \mathrm{X}}$ mutated plasmids were transformed into TKW14 by electroporation using a GenPulser with Pulse Controller (BioRad, Hercules, CA). The pRS314 and pTKERG7RSWT plasmids were also transformed as negative and positive controls, respectively. The resulting transformants were selected for growth on SD+Ade+Lys+His+Met+Ura+hemin+G418+Erg plates, and then reselected on 
SD+Ade+Lys+His+Met+Ura+hemin+G418+5-FOA plates, to determine the complementation effect on ERG7 deficiency, as described previously., Transformants were grown in SD+Ade+Lys+His+Met+Ura+hemin+G418 for nonsaponifiable lipid (NSL) extraction and column chromatography.

Analysis of Extracts from ERG $7^{\mathrm{F} 45 \mathrm{X}}$ Site-Saturated Mutants. The NSL extracts of TKW14[pERG $7^{\mathrm{F} 445 \mathrm{X}}$ ] site-saturated mutants were fractionated by silica gel column chromatography using a 19:1 hexane/ethyl acetate mixture. The fractions were assayed by gas-chromatography-mass spectrometry (GC-MS) and examined for the triterpenoid products with a molecular mass of $\mathrm{m} / \mathrm{z}=426$. GC analyses were performed with an Agilent 6890N chromatograph equipped with a DB-5HT column (30 m x $0.25 \mathrm{~mm}$ I.D., $0.25 \mu \mathrm{m}$ film; oven gradient at $50^{\circ} \mathrm{C}$ for $2 \mathrm{~min}$, and then $20^{\circ} \mathrm{C}$ per min until $300^{\circ} \mathrm{C}$, held at $300^{\circ} \mathrm{C}$ for $20 \mathrm{~min}, 300^{\circ} \mathrm{C}$ injector; $250^{\circ} \mathrm{C}$ interface; $1 / 40$ split ratio using helium carrier gas at 13 psi column head pressure). GC/MS was performed on an Agilent 6890N chromatograph coupled to an Agilent 5973network mass selective detector $\left(\mathrm{EI}^{+}, 70 \mathrm{eV}, 180^{\circ} \mathrm{C}\right.$ ion source temperature, 30-650 amu scan range).

Chemical shifts of (13 $\alpha H)$-isomalabarica-14(26),17E,21-trien-3 $\beta$-ol: Chemical shifts were referenced to $\mathrm{Si}\left(\mathrm{CH}_{3}\right)_{4}$ and are generally accurate to $+0.01 \mathrm{ppm}$. ${ }^{1} \mathrm{H}$ NMR (600 MHz, $\mathrm{CDCl}_{3}$ ): $\delta$ 5.11-5.08 (m, 2H, H-17 and H-21), 4.84 and 4.60 (s and s, $1 \mathrm{H}$ and 1H, $\mathrm{CH}_{2}-14$ ), 3.23 (dd, $J=11.7,5.0 \mathrm{~Hz}, \mathrm{H}-3 \alpha$ ), 2.17-2.11 (m, 2H, H-16), 2.07 (d, $J=8.9 \mathrm{~Hz}, 1 \mathrm{H}, \mathrm{H}-13 \alpha), 2.06$ (m, 2H, $\left.\mathrm{CH}_{2}-20\right), 2.04$ (m, 1H, H-16), 1.98-1.94 (m, 3H,

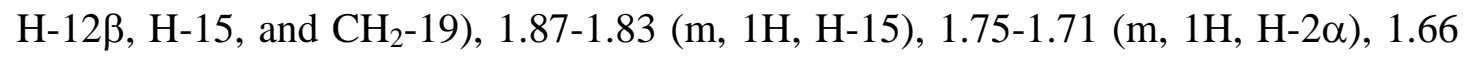

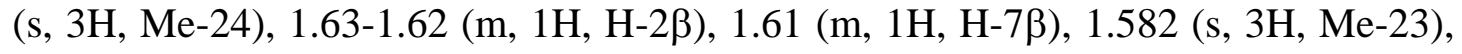
1.579 (s, 3H, Me-25), 1.55 (s, 1H, H-12 $\alpha$ ), 1.54-1.51 (5H, H-5 $\alpha, \mathrm{H}-9 \beta, \mathrm{H}-11 \beta, \mathrm{H}-6 \alpha$ ), 


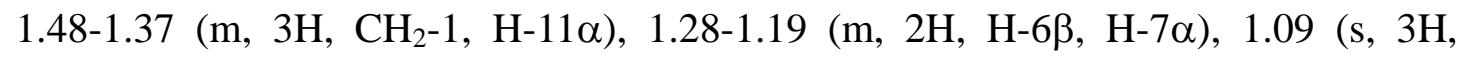
Me-27), 0.96 (s, 3H, Me-29), 0.93 (s, 3H, Me-28), 0.75 (s, 3H, Me-30); ${ }^{13} \mathrm{C}$ NMR (149.90 MHz, $30 \mathrm{mM}$ solution in $\mathrm{CDCl}_{3}, 25^{\circ} \mathrm{C}$ ) $\delta 15.81$ (C-30), 16.01 (C-25), 17.68 (C-23), 18.55 (C-6), 20.74 (C-11), 23.11 (C-28), 25.69 (C-24), 26.53 (C-16), 26.72 (C-20), 28.36 (C-12), 29.08 (C-29), 29.17 (C-2), 29.64 (C-27), 31.93 (C-7), 34.27 (C-1), 35.27 (C-10), 39.06 (C-4), 39.22 (C-15), 39.69 (C-19), 44.79 (C-8), 46.70 (C-5), 52.31 (C-9), 56.47 (C-13), 79.53 (C-3), 109.14 (C-26), 124.15 (C-17), 124.37 (C-21), 131.29 (C-22), 135.14 (C-18), 155.08 (C-14).

Chemical Shifts of 9 $\beta$-lanosta-7,24-dien-3 $\beta$-ol 3-acetate: ${ }^{13} \mathrm{C}$ NMR (150.74 MHz, 20 $\mathrm{mM}$ solution in $\mathrm{CDCl}_{3}, 25^{\circ} \mathrm{C}$ ) $\delta 17.47$ (C-28), 17.62 (C-27), 18.31 (C-21), 21.32 ( $\underline{\left.\mathrm{CH}_{3} \mathrm{CO}-\right),} 22.91$ (C-11), 22.91 (C-6), 23.60 (C-18), 24.31 (C-2), 24.47 (C-19), 25.04 (C-23), 25.71 (C-26), 28.61 (C-16), 28.74 (C-29), 30.53 (C-30), 33.37 (C-15), 35.17 (C-1), 35.22 (C-12), 35.72 (C-10), 35.99 (C-20), 36.10 (C-22), 37.78 (C-4), 43.48 (C-13), 48.39 (C-9), 48.55 (C-5), 52.75 (C-14), 53.48 (C-17), 81.11 (C-3), 121.11 (C-7), 125.18 (C-24), 130.92 (C-25), 149.08 (C-8), 171.00 ( $\left.\mathrm{CH}_{3} \mathrm{CO}-\right)$. Chemical shifts $( \pm 0.02 \mathrm{ppm})$ were referenced to the $\mathrm{CDCl}_{3}$ signal at $\delta 77.0 \mathrm{ppm} .{ }^{13} \mathrm{C} \mathrm{NMR}$ analyses yield spectra consistent with the data published in the literature. ${ }^{5}$

Lanosteryl acetate. ${ }^{13} \mathrm{C}$ NMR (150.68 MHz, $30 \mathrm{mM}$ solution in $\left.\mathrm{CDCl}_{3}, 25{ }^{\circ} \mathrm{C}\right) \delta$ 15.73 (C-18), 16.51 (C-29), 17.62 (C-27), 18.10 (C-6), 18.61 (C-21), 19.16 (C-19), 20.97 (C-11), 21.32 ( $\left.\underline{\mathrm{CH}}_{3} \mathrm{CO}-\right), 24.15$ (C-2), 24.22 (C-30), 24.90 (C-23), 25.71(C-26), 26.35 (C-7), 27.89 (C-28), 28.18 (C-16), 30.80 (C-15), 30.94 (C-12), 35.24 (C-1), 36.24 (C-20), 36.32 (C-22), 36.87 (C-10), 37.78 (C-4), 44.45 (C-13), 49.78 (C-14), 50.35 (C-17), 50.47 (C-5), 80.91 (C-3), 125.22 (C-24), 130.89 (C-25), 134.21 (C-9), 134.46 (C-8), 171.00 ( $\left.\mathrm{CH}_{3} \underline{\mathrm{CO}}-\right)$. Chemical shifts $( \pm 0.02 \mathrm{ppm})$ were referenced to 
the $\mathrm{CDCl}_{3}$ signal at $\delta 77.0 \mathrm{ppm} .{ }^{13} \mathrm{C}$ NMR analyses yield spectra consistent with the data published in the literature. ${ }^{2}$

\section{References:}

1. Wu, T. K.; Yu, M. T.; Liu, Y. T.; Chang, C. H.; Wang, H. J.; Diau, E. W. G.. Org. Lett. 2006, 8, 1319-1322.

2. Wu, T. K.; Liu, Y. T.; Chang, C. H.; Yu, M. T.; Wang, H. J. J. Am. Chem. Soc. 2006, 128, 6414-6419.

3. Wu, T. K.; Chang, C. H. ChemBioChem 2004, 5, 1712-1715.

4. Wu, T. K.; Liu, Y. T.; Chang, C. H. ChemBioChem 2005, 6, 1177-1181.

5. Herrera, J. B. R.; Wilson, W. K.; Matsuda, S. P. T. J. Am. Chem. Soc. 2000, 122, 6765-6766. 
${ }^{1} \mathrm{H}-\mathrm{NMR}$ spectrum of lanosteryl acetate and $9 \beta$-lanosta-7,24-dien-3 $\beta$-ol 3-acetate mixture

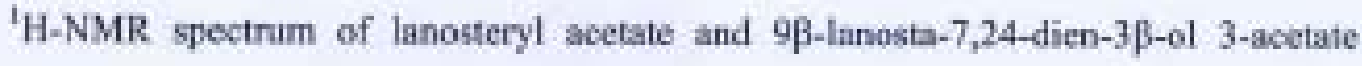
mixture

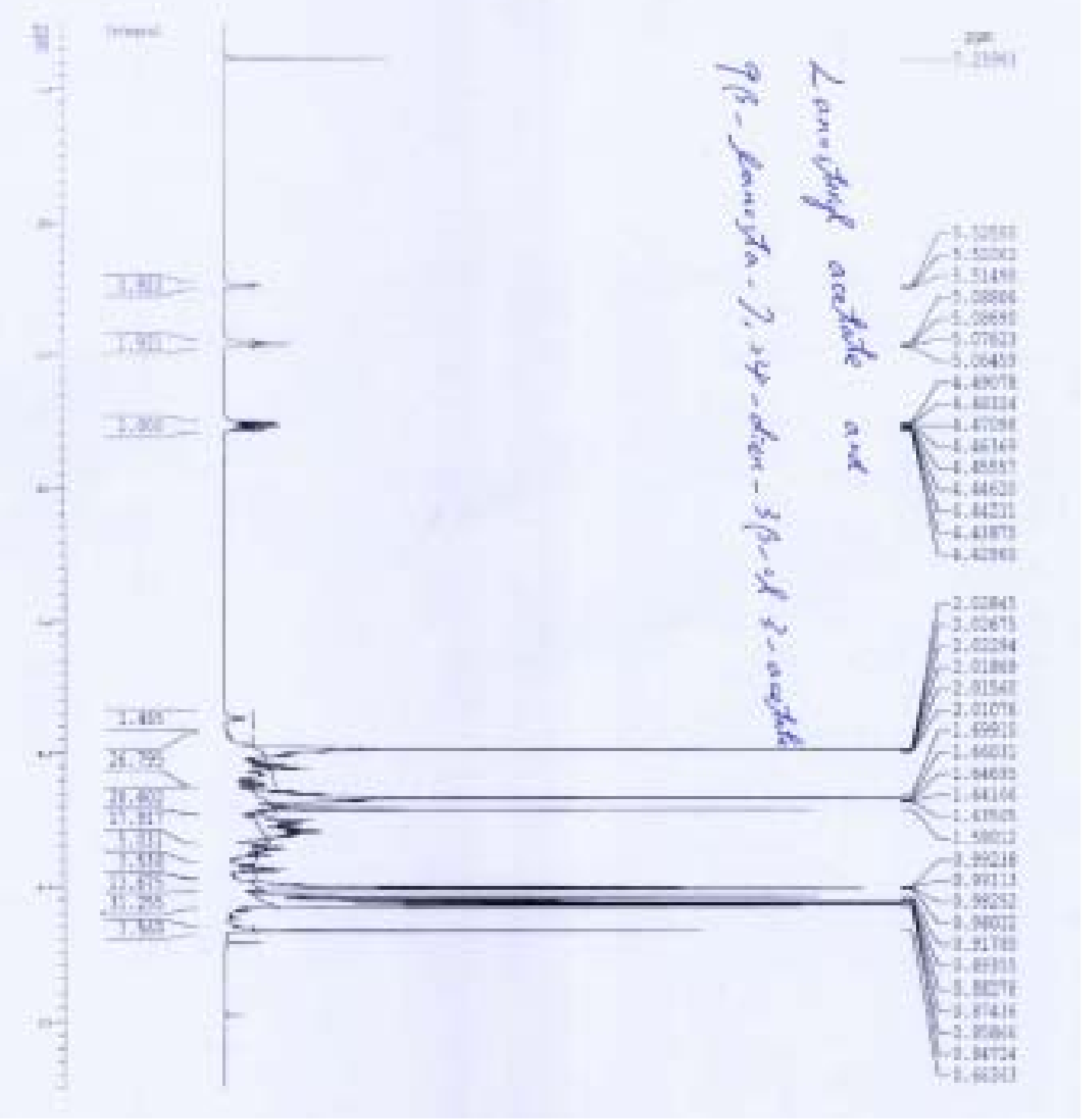


${ }^{13} \mathrm{C}-\mathrm{NMR}$ spectrum of lanosteryl acetate and 9 $\beta$-lanosta-7,24-dien-3 $\beta$-ol 3-acetate mixture

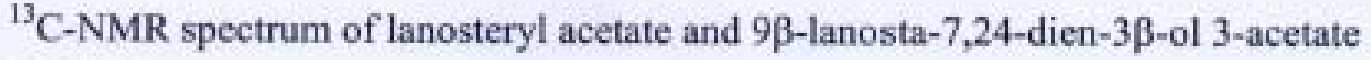 mixture

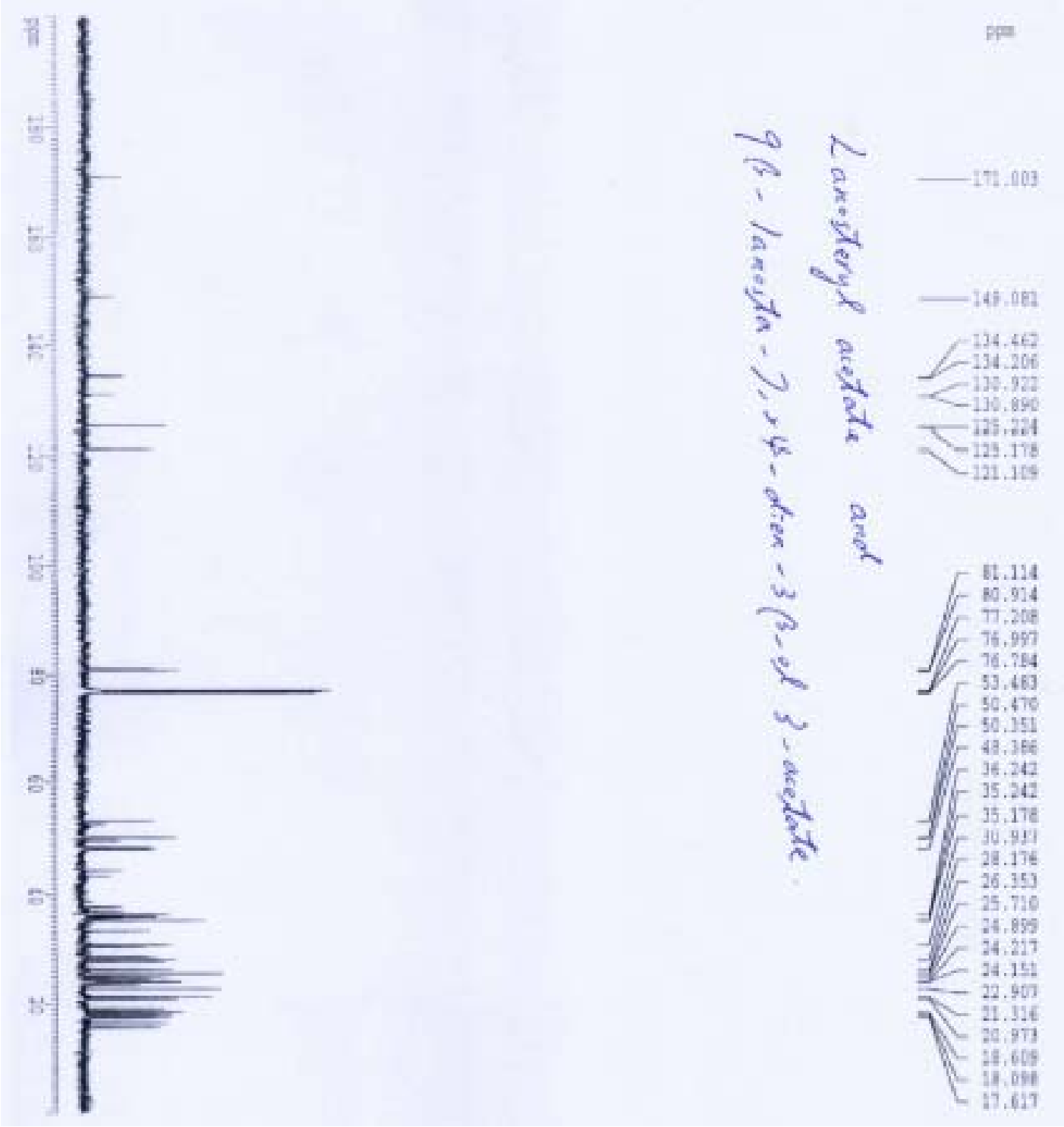


DEPT spectrum of lanosteryl acetate and 9 $\beta$-lanosta-7,24-dien-3 $\beta$-ol 3-acetate mixture

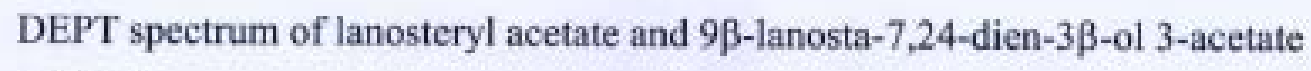
mixture
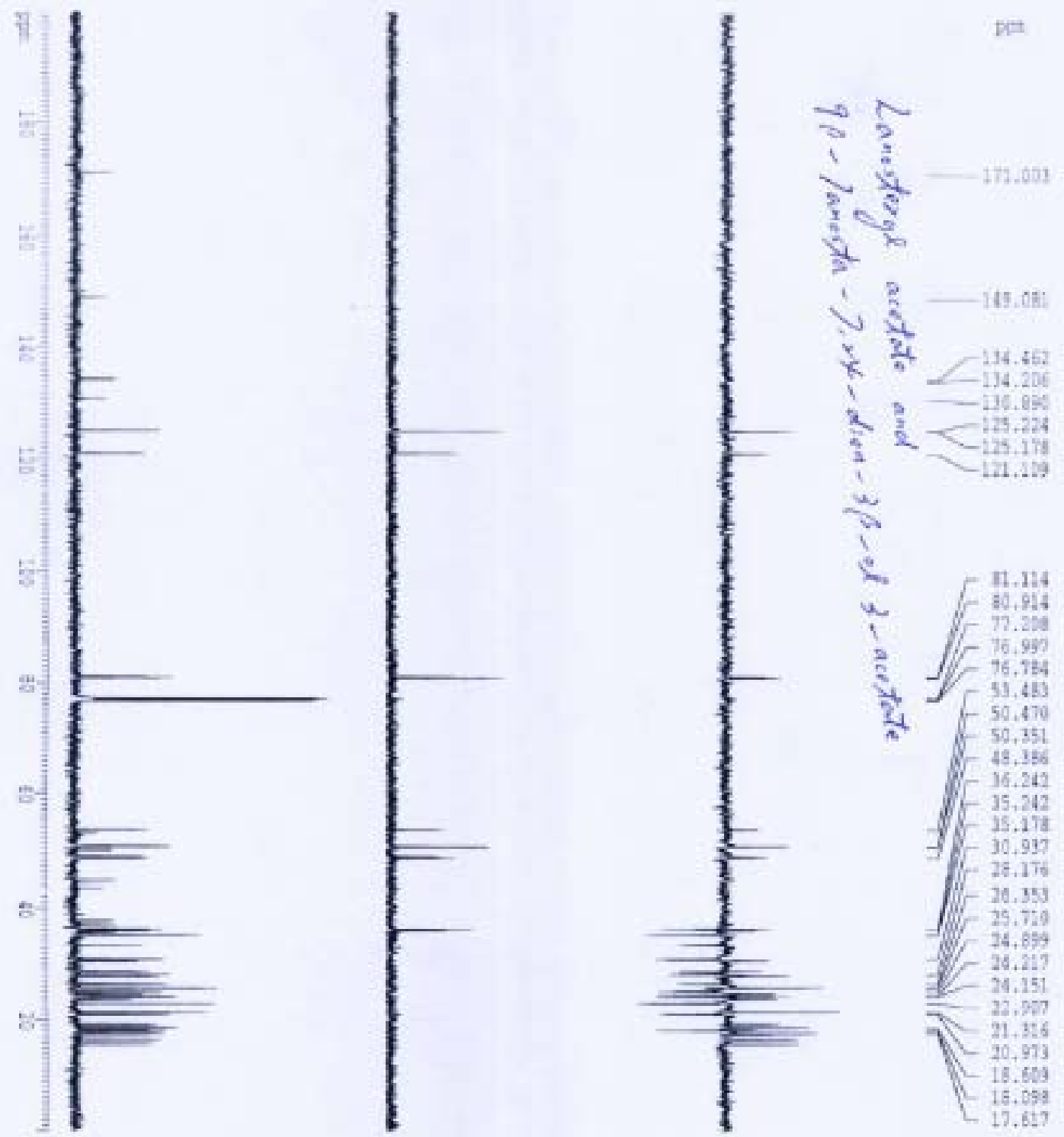
Expanded DEPT spectrum of lanosteryl acetate and $9 \beta$-lanosta-7,24-dien-3 $\beta$-ol 3-acetate mixture (1)

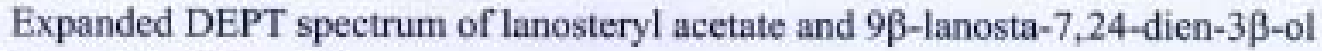
3-acetate mixture (1)
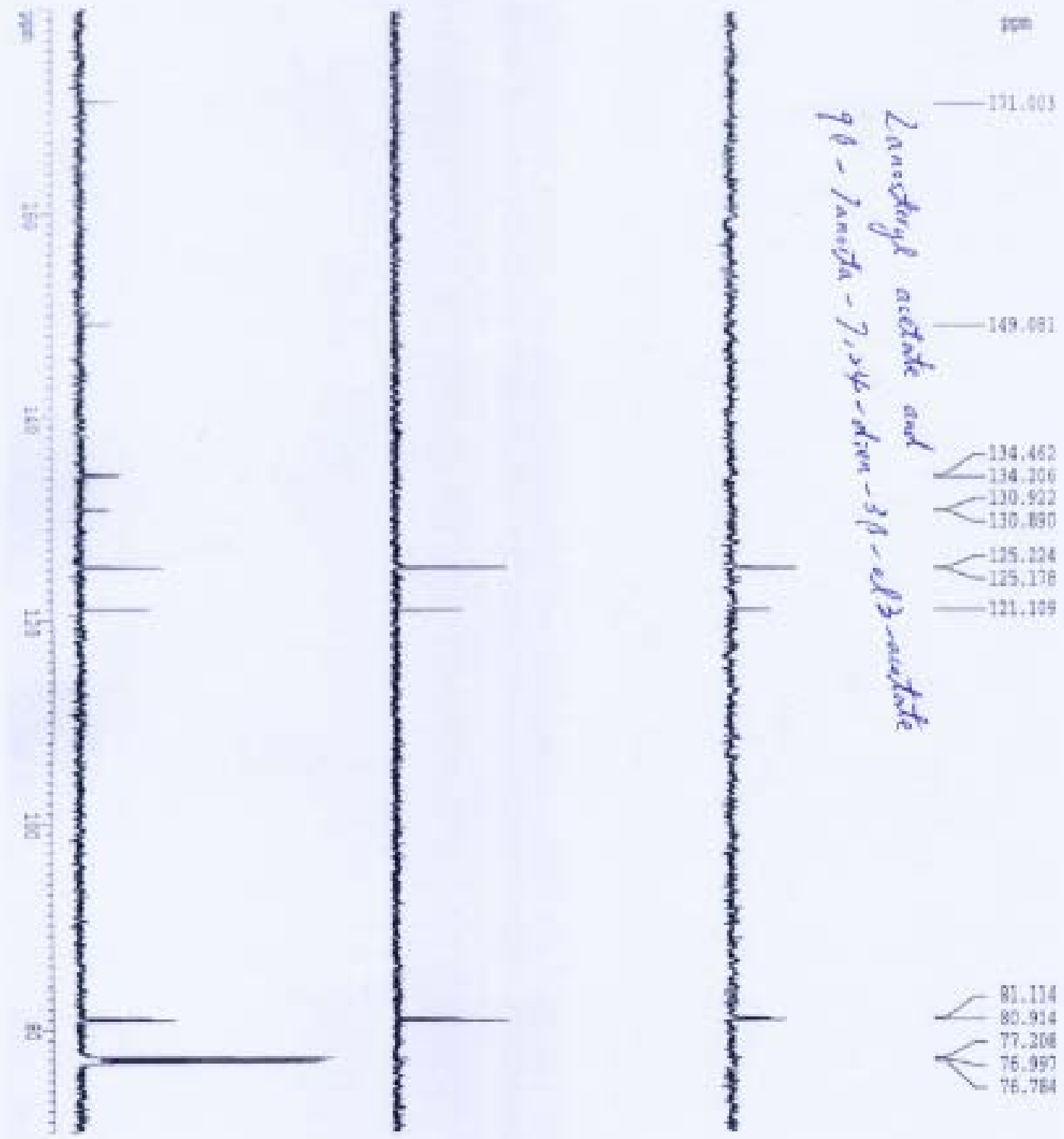
Expanded DEPT spectrum of lanosteryl acetate and 9 $\beta$-lanosta-7,24-dien-3 $\beta$-ol 3-acetate mixture (2)

Expanded DEPT spectrum of lanosteryl acetate and 9 $\beta$-lanosta-7,24-dien-3 $\beta$-ol 3-acetate mixture (2)
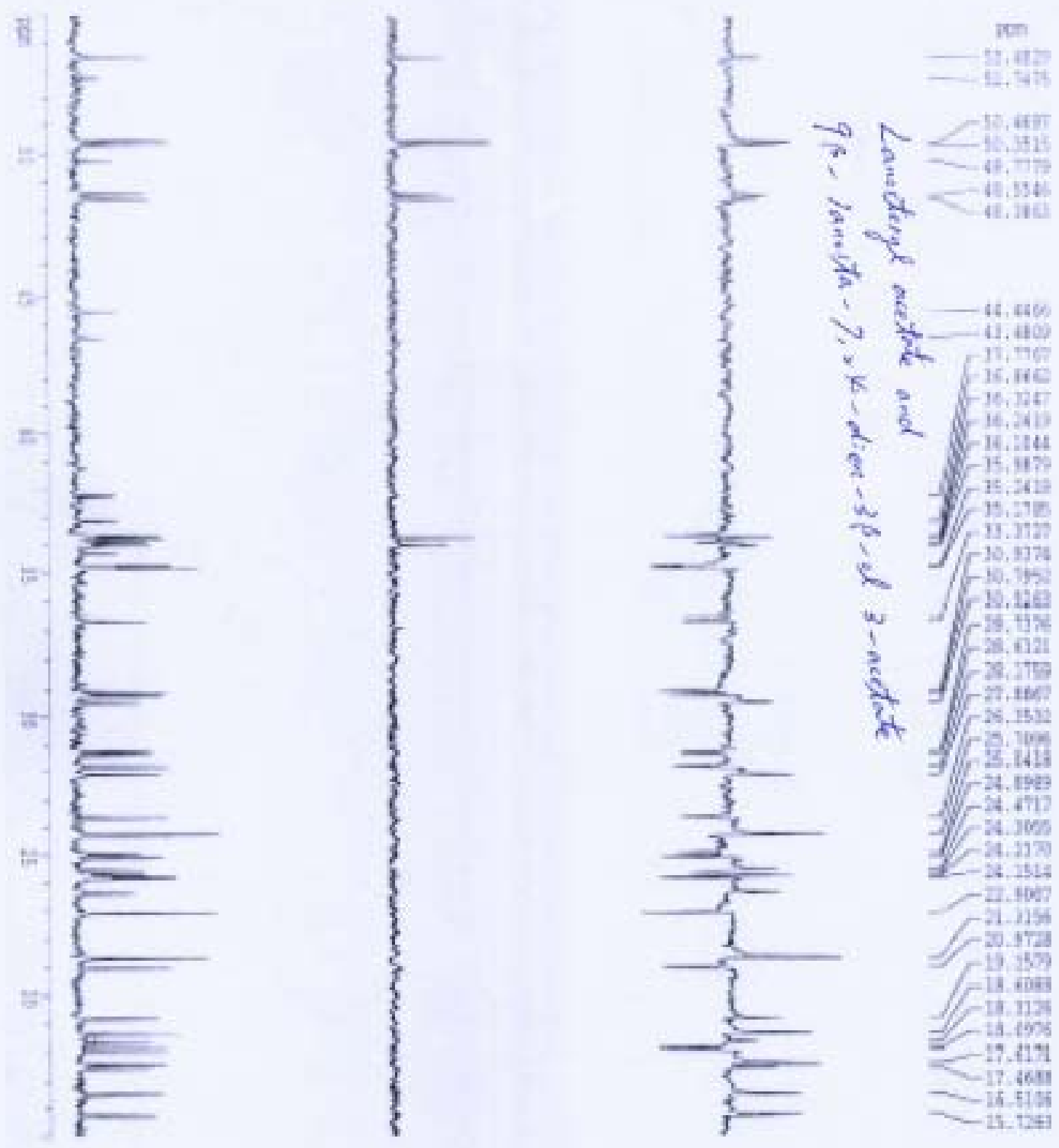
Expanded DEPT spectrum of lanosteryl acetate and $9 \beta$-lanosta-7,24-dien-3 $\beta$-ol 3-acetate mixture (3)

Expanded DEPT spectrum of lanosteryl acetate and 9 $\beta$-lanosta-7,24-dien-3 $\beta$-ol 3-acetate mixture (3)
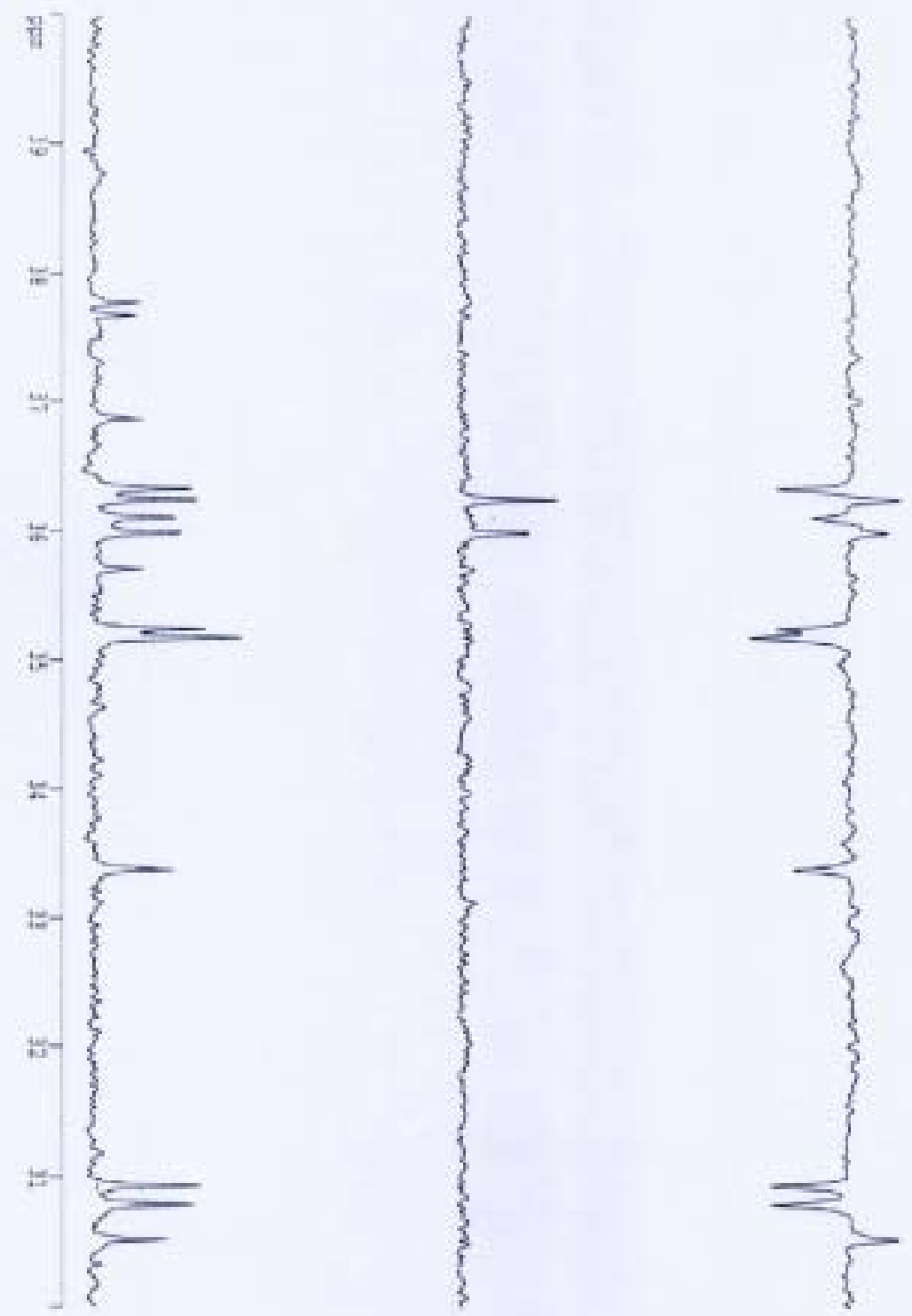

pgan
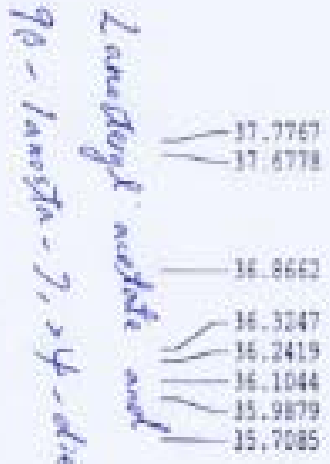

3

$=-35.2418$

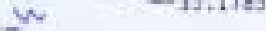

3.

e.

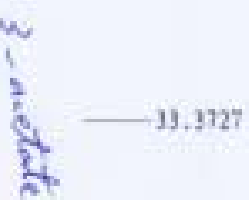


Expanded DEPT spectrum of lanosteryl acetate and $9 \beta$-lanosta-7,24-dien-3 $\beta$-ol 3-acetate mixture (4)

Expanded DEPT spectrum of lanosteryl acetate and $9 \beta$-lanosta-7,24-dien-3 $\beta$-ol 3-acetate mixture (4)
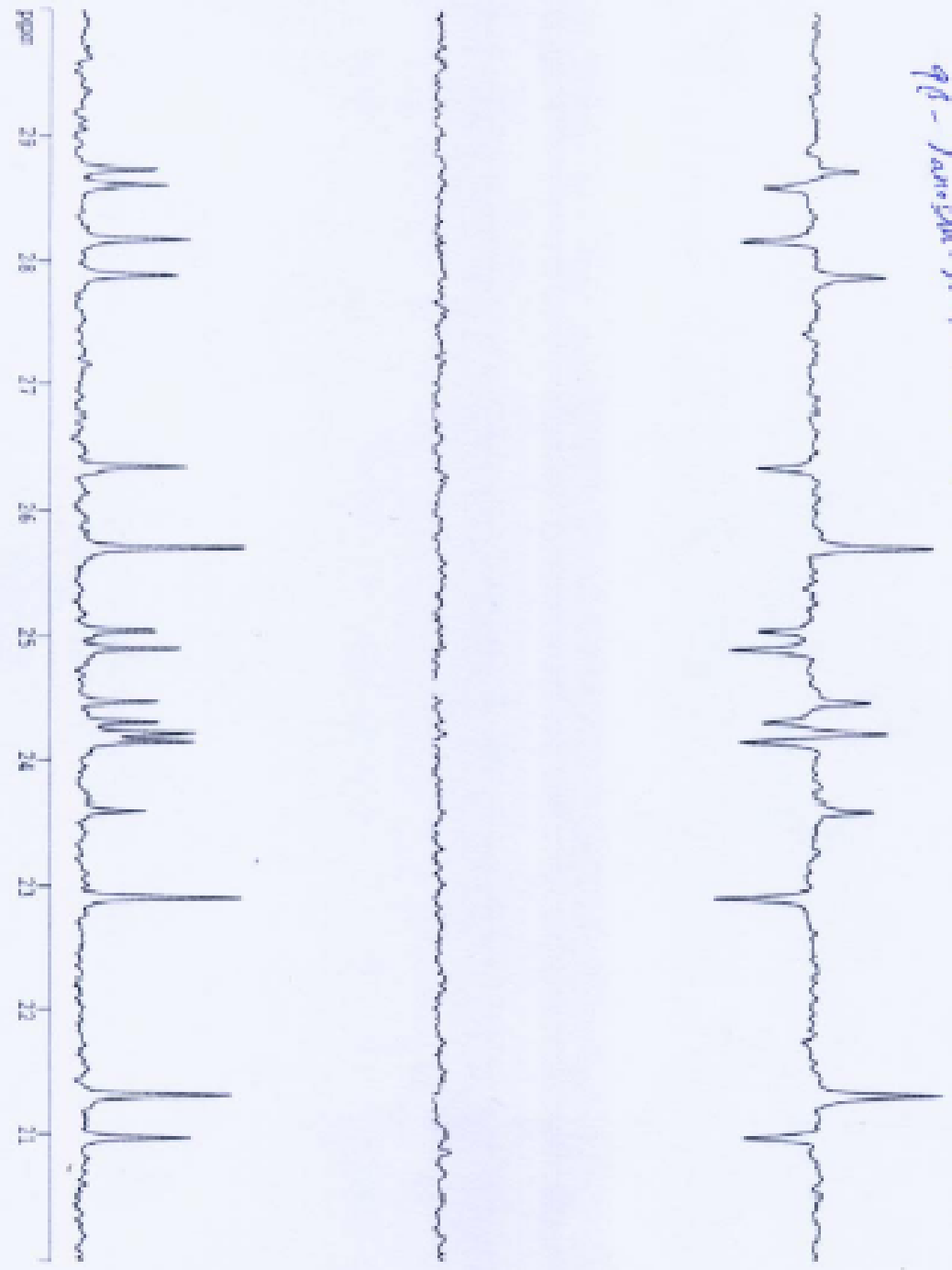

jele

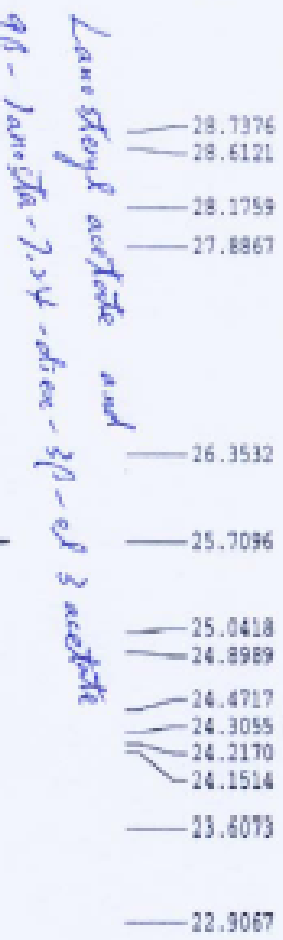

$-21.3156$ 